\title{
LEIOMYOSARCOMA OF THE RENAL VEIN
}

\author{
GUSTAVO C. LEMOS, OMAR R. EL HAYEK, MARCELO APEZZATO
}

Albert Einstein Jewish Hospital, São Paulo, SP, Brazil

\begin{abstract}
Leiomyosarcoma of the renal vein is a rare tumor of complex diagnosis. We presented a case of renal vein leiomyosarcoma detected in a routine study. The primary treatment was complete surgical removal of the mass. In cases where surgical removal is not possible the prognosis is poor, with high rates of local recurrence and distant spread.
\end{abstract}

Key words: kidney; renal veins; muscle neoplasms; leiomyosarcoma

Int Braz J Urol. 2003; 29: 43-4

\section{INTRODUCTION}

There are approximately 30 cases of leiomyosarcoma of the renal vein reported in literature. They affect primarily the inferior vena cava in more than $50 \%$ of the cases (1). Pre-operative diagnosis is difficult due to its low incidence, and since it presents a slow growing rhythm and nonspecific symptoms. We present a case of primary leiomyosarcoma of the left renal vein.

\section{CASE REPORT}

Patient with 47 years, male, white came to the urologist to routine exam. In physical examination identified a mass in left flank, painless and non mobile, by palpation. An abdominal ultrasound showed a tumor without not related to renal parenchyma and excretory route but in close contact with the renal vein (Figure-1).

During the surgery we found that the tumor had its origins in the renal vein and the patient was submitted to radical left nephrectomy (Figure-2). The histological study disclosed renal vein leiomyosarcoma.

\section{DISCUSSION}

Leiomyosarcoma is an uncommon soft tissue tumor, generally occurring in myometrium, respiratory tract and retroperitoneal organs. It seldom originates in vascular structures, and the inferior vena cava responds for more than $50 \%$ of the cases (2). Leiomyosarcoma of the inferior vena cava was first described in 1871 (1), and its diagnosis and treatment is still challenging. These tumors attain women over 30 years in $85 \%$ of the cases (2). More frequently they are left-sided (64\%). Symptoms are nonspecific, such as mild lumbar and abdominal pain, and wasting. Hematuria and palpable mass are rare (2).

Until the 80's, approximately $50 \%$ of the cases were autopsy findings. Presently they are incidentally found in routine studies (2). Clinical context and ultrasound and computed tomography studies are nonspecific and do not allow an adequate differential diagnosis with other retroperitoneal solid tumors (3).

Leiomyosarcoma spread occurs primarily by local extension. Through hematogenous spread it affects liver (25\%), lungs (63\%), bones (19\%) and, less frequently, lymph nodes (3). At diagnosis, approxi- 


\section{LEIOMYOSARCOMA OF THE RENAL VEIN}

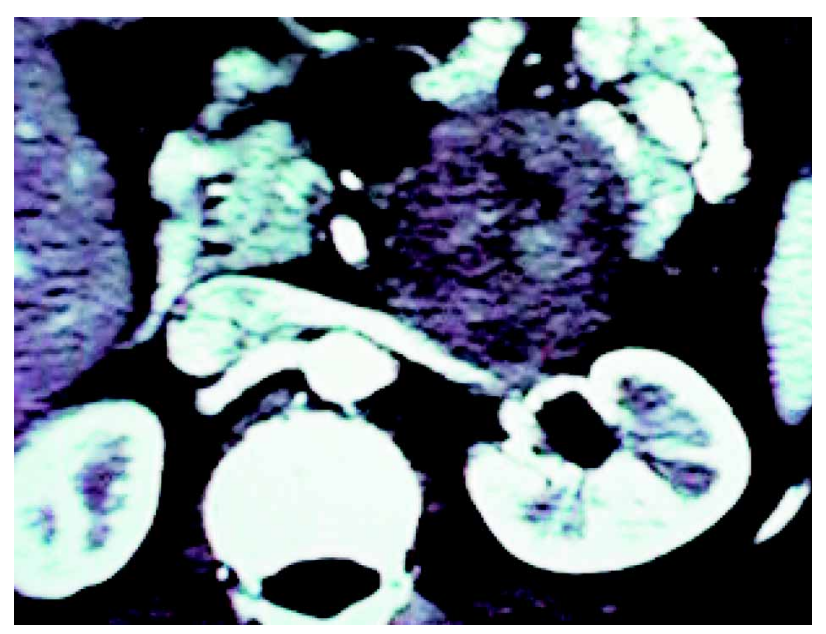

Figure 1 - Abdominal computed tomography showing a bulky mass near the left renal hilum, invading the renal vein.
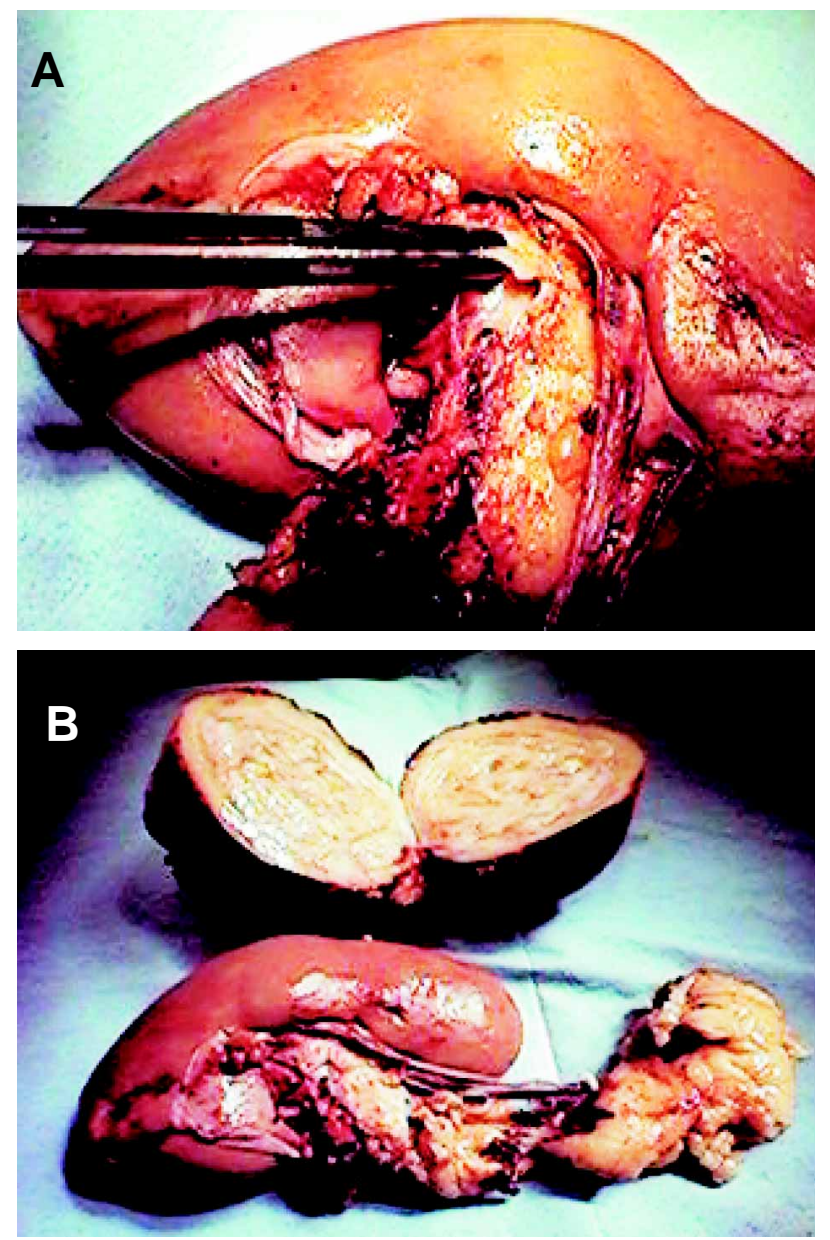

Figure 2 - Surgical specimen. A) - Place of tumor origin near the left renal vein wall. B) - Macroscopic view of the solid mass. mately half of the cases present metastatic disease or are nonresectable due to local invasion, presenting therefore a poor prognosis (3).

The best treatment for leiomyosarcoma is surgery with total tumor removal. This is the option that offers the best chances of local control and 5 years survival. Studies performed at Memorial Sloan Kettering, New York, showed that the major prognostic factor is total surgical resection. When it is complete, 5 years survival free of disease is of approximately $60 \%$, vs. just 30 to $35 \%$ when it is partial. Once total removal is performed, major prognostic factor becomes histological grade, with 5 years free of disease survival of 90 to $95 \%$ for low grade tumors, and of 30 to $35 \%$ for high grade tumors.

Radiotherapy and adjuvant chemotherapy have limited effects due to toxicity on contiguous structures. Adjuvant therapy is generally used to high grade tumors, with partial resection (3).

\section{REFERENCES}

1. Bhathena D, Vasquez M: Primary renal vein leiomyosarcoma. Cancer 1972; 30: 541-4.

2. Brandes SB, Chelsky MJ, Petersen RO, Greenberg RE: Leiomyosarcoma of the renal vein. J Surg Oncol. 1996; 63: 195-200.

3. Bevilacqua RG, Rogatko A, Hadju SI, Brennan MF: Prognostic factors in primary retroperitoneal soft-tissue sarcomas. Arch Surg. 1991; 126: 328-34.

Received: August 12, 2002 Accepted after revision: January 9, 2003 